\title{
Proton antiproton collisions at a finite crossing angle in the SPS
}

\author{
K. Cornelis, W. Herr and M. Meddahi \\ CERN-SL CH-1211 Geneva 23
}

\begin{abstract}
Future high luminosity hadron colliders such as the LHC at CERN and the SSC will have many bunches and therefore require a non-zero crossing angle to avoid multiple bunch crossings outside the interaction point. Long range beam-beam interactions as well as the possible excitation of synchrobetatron resonances due to this crossing angle have been studied theoretically and with computer simulations. However, very little experimental data is available on the effect of a crossing angle in ladron colliders with bunched beams. The electrostatic separators of the CERN SPS used to separate the orbits in its normal operation as a proton-antiproton collider were used to establish a horizontal crossing angle in one of the interaction regions. We have studied the effect of the crossing angle on the bcam dynamics to compare with the expectations from theory and to identify possible limitations for future high luminosity colliders, in particular for the Large Hadron. Collider currently under study at CERN
\end{abstract}

\section{Introduction}

At the LHC a very high luminosity in the order of $10^{34} \mathrm{~cm}^{-2} \mathrm{~s}^{-1}$ is expected [1] and this requires a large number of bunches: for the design luminosity of 1.7 $10^{34} \mathrm{~cm}^{-2} \mathrm{~s}^{-1}$ more than 4000 bunches are necessary. These bunches are normally separated in two vacuum chambers but near the experimental interaction points they share a common beam pipe. It is therefore unavoidable that there will be always a certain number of bunches simultaneously in the same vacuum chamber leading to long range beam-beam interactions. These long range interactions have been studied previously [2] and it has been shown that a crossing angle of $\alpha=200 \mu \mathrm{rad}$ is required to reduce the tune spread due to the long range interactions in the high luminosity intersection regions. However, a finite crossing angle can excite synchro-betatron resonances since the beam-beam interaction depends on the longitudinal position of the particle in the bunch $[3,4]$ and therefore couples the transverse and longitudinal planes. Simulations have been performed for the LHC [j] to test the stability of particles under these conditions and it was shown that the non-zero crossing angle probably has no detrimental effect on the beam.

During the last collider run of the SPS with protons and antiprotons in 1990 it was possible to study the effect of a crossing angle experimentally for a hadron collider. In this report we shall discribe the experiment and present the results and a short discussion of the implications.

\section{Experimental procedure}

For the operation of the SPS as a proton antiproton collider with $6 \times 6$ bunches it was necessary to separate the two beams in most of the ring to avoid parasitic collisions which would effect the beam life time. The schematic layout is shown in Fig.1.

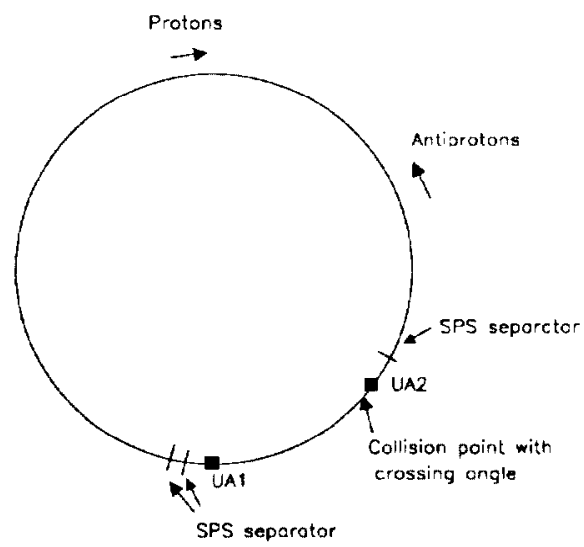

Figure 1: Schematic view of SPS separation scheme

For this purpose three electrostatic separators are used to produce a horizontal pretzel orbit in $5 / 6$ of the ring and only at the experiments and in the middle between the experiments the bunches collide head-on [6].

To successfully collide protons and antiprotons with a finite horizontal crossing angle in one of the interaction regions several constraints have to be met simultaneously:

- The bunches should collide only in the interaction region used for the experiment to avoid a large total tune shift and complicated spectra.

- The crossing angle should be variable over a large range of values, i.ie zero to several hundred $\mu \mathrm{rad}$

- The separation at the interaction point with the crossing angle should be zero.

To simplify the problem we have used only one bunch in each beam, i.e. we have to deal with two collision points only. The above problem can be solved if three independent separator units are available. In order to get to very large crossing angle above $500 \mu \mathrm{rad}$ we had to reduce the high voltage gap in some of the tanks to increase the field for the separation. By these means we obtained crossing angles up to $600 \mu \mathrm{rad}$ (full crossing angle). The theoretical values of the crossing angle were verified with orbit measurements with and without the electrostatic separators. 


\section{Parameters for the experiment}

The bunch length in the SPS is in the order of 3 ns (4 $\sigma)$. The emittances for the experiments varied by a factor of three since some of the experiments were done after a normal physics run and the beams were already diluted after 10 hours of collisions. Two dedicated experiments were done with fresh beams with smaller emittances (normalised emittance $\approx 10 \pi 10^{-6} \mathrm{~m}$ rad) for both particle types. For the nominal emittances $\left(4 \sigma \gamma^{2} / \beta\right)$ and $\approx 10^{11}$ particles per bunch the linear beam-beam tune shift $\xi$ is in the order of $\approx 0.005$, i.e. 1.5 times larger than expected for the LHC.

Another important parameter is the characteristic angle, which is the crossing angle at which the ratio between the longitudinal and transverse dimensions is such that the ends of the bunches separate when the bunch centres collide. The ratio $\alpha \sigma_{s} / 2 \sigma_{x, z}$ should be smaller than one to avoid a significant reduction of the available luminosity and strong excitation of synchro-betatron resonances. The longitudinal and transverse beam sizes arc expressed as the r.m.s. values $\sigma_{s}$ and $\sigma_{x, z}$ and $\alpha$ is the full crossing angle. For the nominal LHC crossing angle of $200 \mu \mathrm{rad}$ this ratio is $\approx 0.5$. With the above parameters is was possible to test ratios between 0 and about 0.7 , i.e. we could explore the entire interesting range of this parameter.

The working point of the LHC is in the neighbourhood of the 12 th order resonance and in order to explore resonances of comparable strength we studied the behaviour near the 10 th order resonance i.e. for tunes around 26.70 horizontally and 27.69 vertically.

The synchrotron frequency for the SPS is up to around $\approx 180 \mathrm{~Hz}$ corresponding to a synchrotron tune of $\approx 0.004$, i.e. about $2-3$ times the values for the $\mathrm{LHC}$.

\section{Preparation}

For all runs at the end of a normal physics run the unwanted bunches had to be killed and this was done with the SPS damper system [7]. However, the remaining bunches always slightly suffered and their emittances increased, making a scraping of the bunches necessary.

\section{Measurements}

With the very small values for the synchrotron tune $Q_{\boldsymbol{s}}$, one cuuld probably not expect to see individual satellite resonances, but the excitation of synchro-betatron sidebands would appear as a broadening of the non-linear res. onances driven by the head-on beam-beam effect. The expcrimental verification therefore is to scan the horizontal tune across the resonance and measure the beam lifetime with and without the crossing angle. The background signals measured at the experiments are a good and very sensitive measure of the beam lifetime and of possible particle losses. The background rates coming from the proton and antiproton beam can be measured individually. For the experiment we have chosen the UA2 area since the background and luminosity counters of the UA2 experiment were made available in the SPS control room.

The tune was measurcd with the SPS Schottky system and the spectra for both planes were recorded during the entire cxperiment [8] and could be inspected later during the analysis. The emittances were measured using the standard wire scanner and if necessary reduced by scraping.

Synchro-betatron resonances also have an effect on the longitudinal phase space and we have used the pickups for the longitudinal feedback system to look for possible changes in the longitudinal spectrum.

\section{Experimental results}

To compare we have made several scans with a zero crossing angle and in Fig.2 we have plotted such a scan for a crossing angle of $500 \mu \mathrm{rad}$ and head-on crossing, i.e. 0 $\mu \mathrm{rad}$. This value gave a ratio $\alpha \sigma_{s} / 2 \sigma_{x_{1} z} \approx 0.45$ i.e. close to the one expected for the LHC.

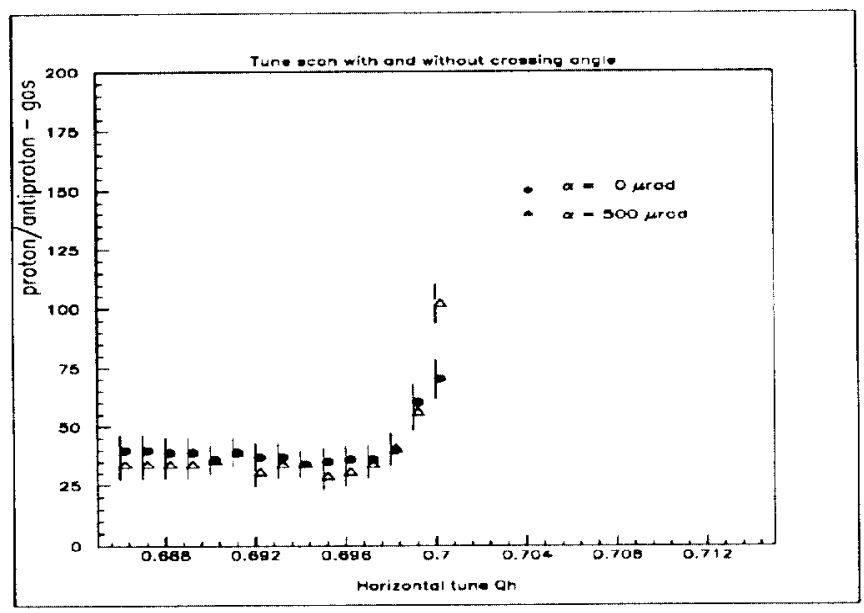

Figure 2: Tune scan with $\alpha=500 \mu \mathrm{rad}$

Taking into account the (purely statistical) errors, no significant change in the behaviour of the background can be seen. In Fig. 3 we have plotted a similar measurement for a smaller crossing angle of $\alpha=250$ r rad.

The absolute values of the background cannot be easily compared because the geometry of the background measurement system changes when a crossing angle is established. It is therefore only meaningful to look for the onset of particle losses when a resonance is approached. For the crossing angle of $250 \mu \mathrm{rad}$ no significant change can be observed as for the previous diagram. The two above measurements where taken during the same run, but later experiments with the same crossing angles gave no different results and are therefore not presented here.

In the last experiment we were able to establish a crossing angle of $600 \mu \mathrm{rad}$ with a relatively small emittance. We could therefore reach values for the ratio $\alpha \sigma_{s} / 2 \sigma_{x, z}$ of more than 0.7. The results are shown in Fig. 4. 


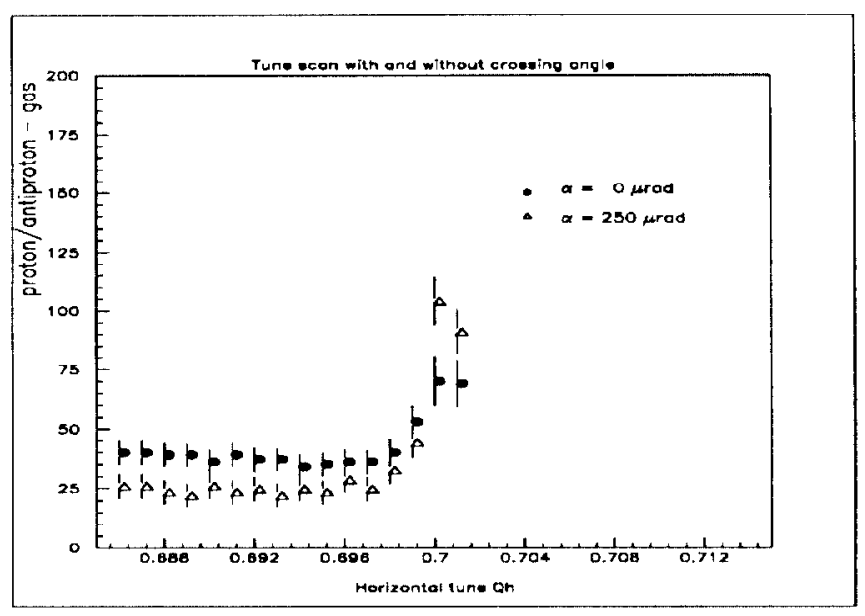

Figure 3: Tune scan with $\alpha=250 \mu \mathrm{rad}$

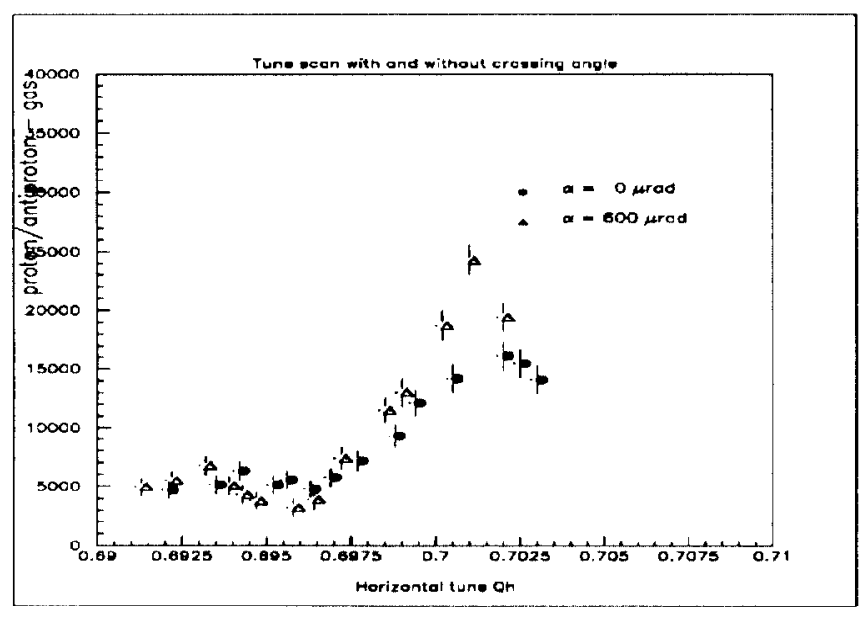

Figure 4: Tune scan with $\alpha=600 \mu \mathrm{rad}$

As it was shown for smaller values of the crossing angle, no significant broadening of the resonances can be seen. The small peak at horizontal tune values around $Q_{h} \approx 0.692$ is the 13 th order resonance $13 Q_{x}=347$ and is stronger in this case as compared with the previous measurements. The Fourier spectra of the horizontal and vertical motion showed no difference to the head-on case.

\section{Conclusion}

We shall try to conclude as follows:

- We have shown that electrostatic separators can be used to establish proton-antiproton collisions at a finite crossing angle in a hadron collider like the SPS.

- Crossing angle up to $600 \mu \mathrm{rad}$ have been reached.

- The comparison between tune scans with and without crossing angle shows no significant differcnce.
- For the nominal LHC parameters (i.e. $\beta^{*}$ function at the interaction point, crossing angle $\alpha$ and separation) it seems unlikely that synchro-betatron resonances significantly affect the beam stability.

- To establish the existence of synchro-betatron resonances in hadron colliders, more experiments are probably necessary.

\section{References}

[1] The LHC study group; Design Study of the Large Hadron Collider (LHC).

CERN/AC/DI/FA/90-06 DRAFT (1991)

[2] W. Herr; Tune shifts and spreads due to the long rangc beam-bcam effcct in the $L H C$.

CERN/SL/90-06 (AP) and LHC Note 119 (1990).

[3] A. Piwinski; Satellite resonances due to beam-beam interaction

IEEE Trans. Nucl. Sci. NS-24, 1408 (1977).

[4] A. Piwinski; Computer simulation of the beam-beam interaction at a crossing angle

IEEE Trans. Nucl. Sci. NS-32, 2240 (1977).

[5] W. Herr; Computer simulations of synchro-betatron resonances induced by a non-zero crossing angle in the LHC. CERN/SL/90-70 (AP) (1990).

[6] K. Cornelis et. al.; Operation of the SPS separation scheme CERN/SPS/87-1 (AMS) (1987).

[7] K. Cornelis, M. Meddahi and R. Schmidt; Experiments on the beam-beam effect in the CERN-SPS in the 1989 collider run. CERN/SPS/AMS/Note 89-13 (1989).

[8] K. Cornelis, M. Meddahi, R. Schmidt and D. Vandeplassche; Experimental study of a beam excitation in the presence of the beam-beam interaction. CERN/SPS/AMS/Note 89-04 (1989). 